Eilers, M. \& Möbus C., Learning the Relevant Percepts of Modular Hierarchical Bayesian Driver Models using a Bayesian Information Criterion, in: Vincent G. Duffy (Ed.), Digital Human Modeling, HCI 2011, Orlando, FL, USA

\title{
Learning the Relevant Percepts of Modular Hierarchical Bayesian Driver Models using a Bayesian Information Criterion
}

\author{
Mark Eilers $^{1,2}$ and Claus Möbus ${ }^{1,2}$ \\ Transportation Systems, Learning and Cognitive Systems \\ OFFIS Institute for Information Technology, C.v.O. University, Oldenburg, Germany \\ http://www.offis.de/, http://www.lks.uni-oldenburg.de/ \\ Mark.Eilers@offis.de,Claus.Moebus@uni-oldenburg.de
}

\begin{abstract}
Modeling drivers' behavior is essential for the rapid prototyping of error-compensating assistance systems. Various authors proposed controltheoretic and production-system models. Based on psychological studies various percepts and measures (angles, distances, time-to-x-measures) have been proposed for such models. These proposals are partly contradictory and depend on special experimental settings. A general computational vision theory of driving behavior is still pending. We propose the selection of drivers' percepts according to their statistical relevance. In this paper we present a new machine-learning method based on a variant of the Bayesian Information Criterion (BIC) using a parent-child-monitor to obtain minimal sets of percepts which are relevant for drivers' actions in arbitrary scenarios or maneuvers.
\end{abstract}

Keywords: Probabilistic Driver model, Bayesian Autonomous Driver model, Mixture-of-Behavior model, Bayesian Real-Time-Control, Machine-Learning, Bayesian Information Criterion, Hierarchical Bayesian Models

\section{Introduction}

The Human or Cognitive Centered Design of intelligent transport systems requires computational models of human behavior and cognition [1, 2]. Particularly the modeling of drivers' behavior is essential for the rapid prototyping of errorcompensating assistance systems [1]. Based on psychological studies [3, 9-11, 13, 20, 21] various percepts and measures (angles, distances, time-to-x-measures) have been proposed for such models. These proposals are partly contradictory and depend on special experimental settings. A general computational vision theory of driving behaviour is still pending.

Due to the variability of human cognition and behavior, the irreducible lack of knowledge about underlying cognitive mechanisms, and irreducible incompleteness of

${ }^{1}$ project Integrated Modeling for Safe Transportation (IMOST) sponsored by the Government of Lower Saxony, Germany under contracts ZN2245, ZN2253, ZN2366

2 project ISi-PADAS funded by the European Commission in the 7th Framework Program, Theme 7 Transport FP7-218552 
Eilers, M. \& Möbus C., Learning the Relevant Percepts of Modular Hierarchical Bayesian Driver Models using a Bayesian Information Criterion, in: Vincent G. Duffy (Ed.), Digital Human Modeling, HCI 2011, Orlando, FL, USA

knowledge about the environment [1] we conceptualize, estimate and implement models of human drivers as probabilistic models: Bayesian Autonomous Driver (BAD) models. In contrast to [21], BAD models don't need to be programmed like traditional simulation software but are condensed and abstracted in an objective manner using machine-learning techniques from human behavior traces.

\section{Bayesian Autonomous Driver Mixture-of-Behaviors Models}

In earlier research [14] we developed a BAD model with Dynamic Bayesian Networks based on the Bayesian Programming approach [1] and on the assumption that a single skill is sufficient for lateral and longitudinal control. Later, we realized that for modeling the complex competence of human drivers a skill hierarchy is necessary. We modified the simple BAD model architecture to a hierarchical modular probabilistic architecture to construct driver models by decomposing complex maneuvers into basic behaviors and vice versa: Bayesian Autonomous Driver Mixture-of-Behaviors (BAD MoB) models [5, 6, 15, 16].

BAD MoB models consist of Gating-, Behavior-Classification-, and Actionmodels. Their functional interaction allow the generation of context dependent driver behavior by sequencing and mixing pure basic behaviors [5, 6] (Fig. 1).

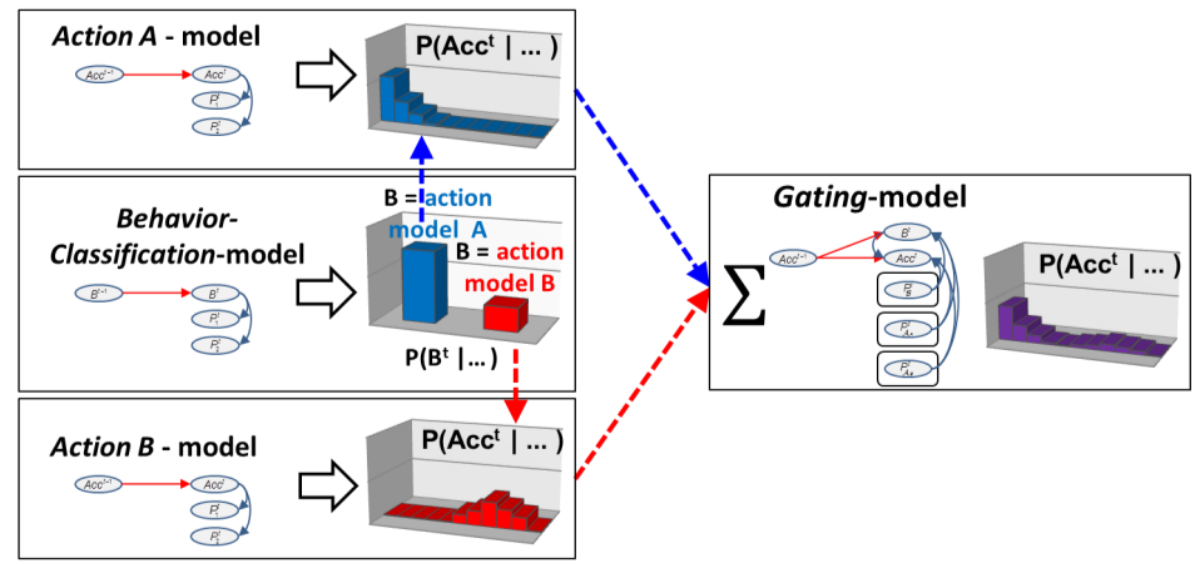

Fig. 1. Exemplary mixing of behaviors in a BAD MoB model assembled from two Action-models, one Behavior-Classification, and one Gating model. The Gating-model calculates a weighted sum over the answers of the two Action-models, according to the appropriateness of their corresponding behaviors, respectively their probabilities or mixing coefficients, inferred by the Behavior-Classification-model.

Based on a skill hierarchy, partitioning complex scenarios into maneuvers and maneuvers into simpler behaviors (cf. Fig. 2), each behavior is modeled by an Actionmodel. This is implemented by a dynamic Bayesian network that realizes the sensormotor schema of the desired behavior. It can be utilized to infer the conditional probability distribution (CPD) of the actions given the former actions and the current percepts: $P\left(\right.$ Actions $^{t} \mid$ Actions $^{t-1}$, Percepts $\left.^{t}\right)$. For each complex scenario (or maneuver) in 
Eilers, M. \& Möbus C., Learning the Relevant Percepts of Modular Hierarchical Bayesian Driver Models using a Bayesian Information Criterion, in: Vincent G. Duffy (Ed.), Digital Human Modeling, HCI 2011, Orlando, FL, USA

the skill hierarchy a Behavior-Classification-model is used to infer the appropriateness of the corresponding simpler maneuvers (or behaviors). A Gatingmodel computes a weighted sum over the inferred CPDs of the Action-models by using the appropriateness of their corresponding behaviors, inferred by the BehaviorClassification-models in the form of mixing coefficients (Fig. 1). By calculating weighted sums over the mixture distributions BAD MoB models are able to combine mixture distributions in a hierarchically manner. Thus these models allow the combination of pure behaviors into more complex maneuvers and maneuvers into scenarios.

BAD MoB models sample random values Actions $^{t}=$ actions $^{t}$ from the inferred CPD P(Actions ${ }^{t} \mid$ Actions $^{t-1}$, Percepts $\left.^{t}\right)$ every $50 \mathrm{~ms}$. These are used as motor commands to autonomously control (simulated) vehicles.

\subsection{Skill hierarchy}

For an experimental BAD MoB in the racing simulation $\mathrm{TORCS}^{3}$, we defined a skill hierarchy of three hierarchical layers. The Racing Scenario was partitioned into the three maneuvers LaneFollowing, CarFollowing and Overtaking. LaneFollowing was partitioned into the behaviors for driving on a straight segment (Straight), through a wide curve (Wide) and through a sharp curve (Sharp), etc. pp. (Fig. 2).

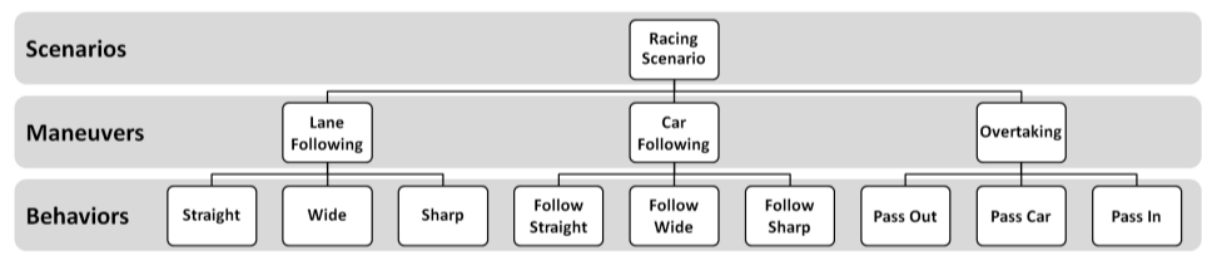

Fig. 2. Skill hierarchy for a racing scenario with three hierarchical layers.

\subsection{Training Phase}

The learning of a BAD MoB model requires time series of human behavior traces. These were obtained from a single driver, who drove several laps on two different racing courses in the TORCS simulation. We recorded approximately 15000 data samples. Each time-stamped data record contained values for 211 discrete random variables (Table 1): two action-variables $A c c^{t}$ and Steer ${ }^{t}$, denoting the position of a combined acceleration and braking pedal and the steering wheel angle, four behaviorvariables representing the partitioning of the task hierarchy (Fig. 2) and a set of 205 time-independent (estimates of distances and angles) and time-dependent perceptvariables (TDPs), similar but not identical to Lee's time-to-x (tau) measures [12, 13, $17]$.

\footnotetext{
${ }^{3} \mathrm{http}: / /$ torcs.sourceforge.net/ (last retrieved 2011-01-31)
} 
Eilers, M. \& Möbus C., Learning the Relevant Percepts of Modular Hierarchical Bayesian Driver Models using a Bayesian Information Criterion, in: Vincent G. Duffy (Ed.), Digital Human Modeling, HCI 2011, Orlando, FL, USA

Table 1. Overview of the two action-variables, four behavior-variables and 205 perceptvariables defined for the foveal and ambient visual channel of the driver [7].

\begin{tabular}{|c|c|c|}
\hline Variable & Range & Description \\
\hline$A c c^{t}$ & $\{0, \ldots, 14\}$ & $\begin{array}{l}\text { Position of a combined acceleration and braking pedal. Ranges from } \\
\text { full braking (0) to full acceleration (14). }\end{array}$ \\
\hline Steer ${ }^{t}$ & $\{0, \ldots, 29\}$ & $\begin{array}{l}\text { Steering wheel angle. Ranges from full turning to the right }(0) \text { to full } \\
\text { turning to the left }(29) \text {. }\end{array}$ \\
\hline$B_{S c}^{t}$ & $\{0, \ldots, 2\}$ & $\begin{array}{l}\text { Represents the maneuvers LaneFollowing, CarFollowing and } \\
\text { Overtaking that compose the Racing Scenario. }\end{array}$ \\
\hline$B_{L F}^{t}$ & $\{0, \ldots, 2\}$ & Represents the LaneFollowing behaviors Straight, Wide and Sharp. \\
\hline$B_{C F}^{t}$ & $\{0, \ldots, 2\}$ & $\begin{array}{l}\text { Represents the CarFollowing behaviors FollowStraight, Follow Wide } \\
\text { and FollowSharp. }\end{array}$ \\
\hline$B_{O T}^{t}$ & $\{0, \ldots, 2\}$ & Represents the Overtaking behaviors PassOut, PassCar and PassIn. \\
\hline$L S^{t}$ & $\{0, \ldots, 20\}$ & Longitudinal speed of the ego-car. \\
\hline$F C A_{5 m}^{t}$ & $\{0, \ldots, 20\}$ & 50 Fixed-Distance Course Angles. \\
\hline$F C A_{10 m}^{t}$ & & $\underset{\perp}{\stackrel{F C}{t}}$ \\
\hline$F C A_{250 m}^{t}$ & & - Course of road \\
\hline
\end{tabular}

$S C A_{0.2 s}^{t}$

$S C A^{t}{ }_{0.4 s}$

$\{0, \ldots, 20\} \quad 50$ Speed-Dependent Course Angles.

${ }_{S C} A^{t}{ }_{10.0 s}$

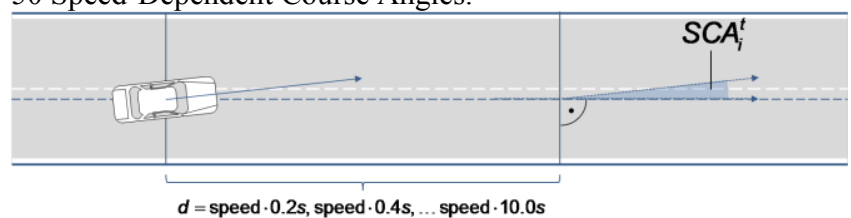

$F L A^{t}{ }_{5 m}$,

$F L A^{t}{ }_{10 m}$

$\ldots$,

$F L A_{250 m}^{t}$

$\{0, \ldots, 20\} \quad 50$ Fixed-Distance Lane Angles.

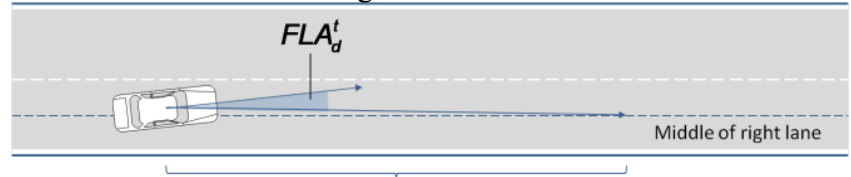

$d=5 m, 10 m, \ldots, 250 m$

$S L A_{0.2 s}^{t}$,

$S L A^{t}{ }_{0.4 S}$

$\ldots$,

$S L A_{10.0 s}^{t}$

$\{0, \ldots, 20\} \quad 50$ Speed-Dependent Lane Angles.

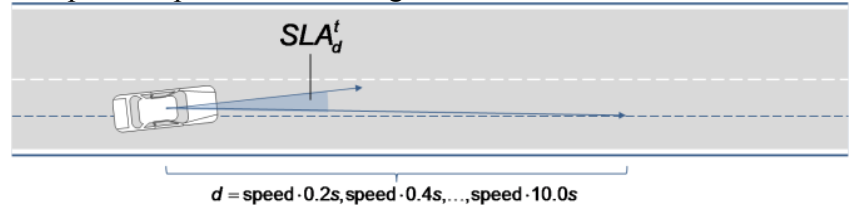

$N C A^{t}$

$\{0, \ldots, 20\} \quad$ Nearest Alter-Car Angle.

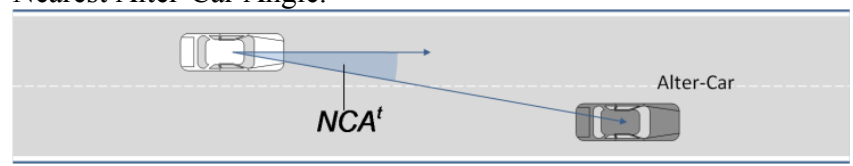


Eilers, M. \& Möbus C., Learning the Relevant Percepts of Modular Hierarchical Bayesian Driver Models using a Bayesian Information Criterion, in: Vincent G. Duffy (Ed.), Digital Human Modeling, HCI 2011, Orlando, FL, USA

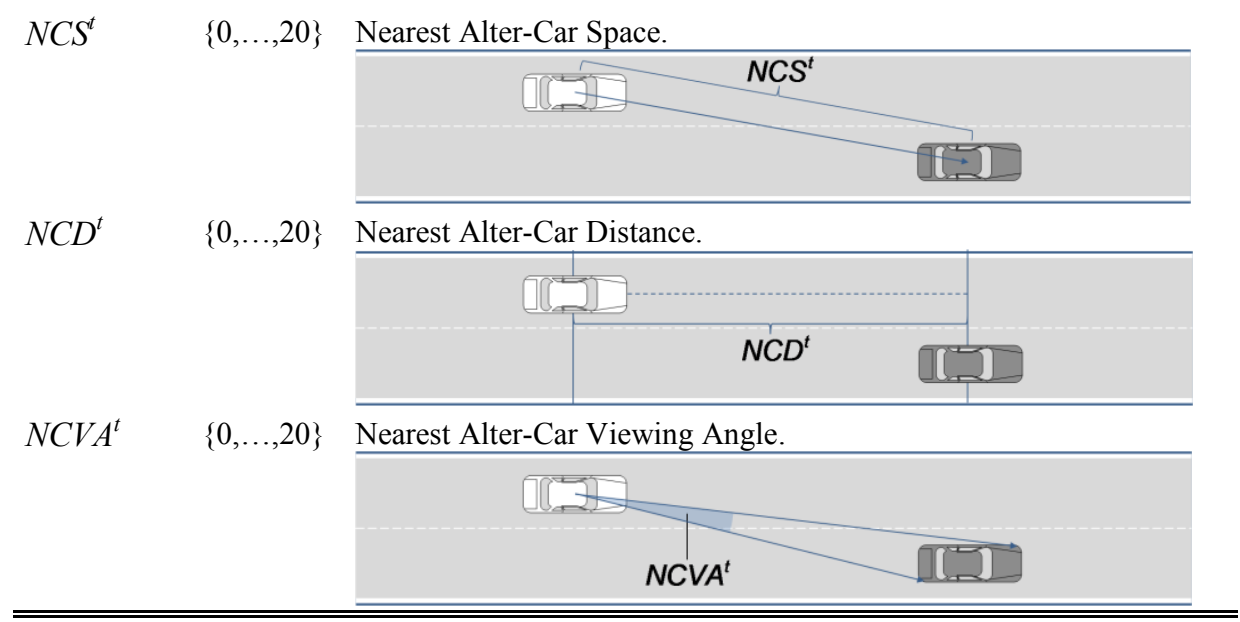

\subsection{Learning of Relevant Peephole Percepts}

Until now the structures of skill hierarchies have to be created manually. But both the graph-structure of Action- and Behavior-Classification-models and the parameters of their (conditional) probability distributions can be obtained by machine-learning methods from time series of human behavior traces. To completely cover the skill hierarchy (Fig. 2) nine Action- and four Behavior-Classification-models have to be learnt $[5,6]$. The structure of four complimentary Gating-models can then be derived automatically from the structure of the Action-and Behavior-Classification-models.

To ensure efficiency for the real-time control of a BAD MoB model, we constrain the structure of Action- and Behavior-Classification-models to dynamic (first order markov) naïve Bayesian Classifiers. For Action-models we further assume the actionvariables $A c c^{t}$ and Steer ${ }^{t}$ to be independent given both of the former actions $A c c^{t-1}$ and Steer ${ }^{t-1}$, and that a percept must not be conditioned on both Acc ${ }^{t}$ and Steer ${ }^{t}$. These assumptions allow the boosting of the inference performance by splitting the intended CPD $P\left(\right.$ Acc $^{t}$,Steer ${ }^{t} \mid$ Acc $^{t-1}$,Steer ${ }^{t-1}$, Percepts $\left.^{t}\right)$ into two independent distributions $P\left(\right.$ Acc $^{t} \mid$ Acc $^{t-1}$, Steer $^{t-1}$, Percepts $\left.^{t}\right)$ for longitudinal and $P\left(\right.$ Steer $^{t} \mid$ Acc $^{t-1}$, Steer $^{t-1}$, Percepts $\left.^{t}\right)$ for lateral control.

Our BAD MoB models rest on the assumption that there is considerable uncertainty about the relevant percepts for realization and classification of natural driving behaviors. So the relevant percepts should be identified during the modeling process. We rely on a step-wise structure-learning technique that exploits the probabilistic foundations of Bayesian driver models and determines the 'peephole' percepts from a universe of hypothetical possible or available percepts based on the Bayesian Information Criterion (BIC) $[4,8,18]$.

\subsubsection{The Parent-Child Bayesian Information Criterion}

The BIC rewards how well a model fits the data while penalizing the model complexity. Let $\delta$ denote a set of $n$ data rows associated with the behavior to be 
Eilers, M. \& Möbus C., Learning the Relevant Percepts of Modular Hierarchical Bayesian Driver Models using a Bayesian Information Criterion, in: Vincent G. Duffy (Ed.), Digital Human Modeling, HCI 2011, Orlando, FL, USA

generated by an Action-model $\pi_{\mathrm{A}}$ or the mixture of behaviors to be classified by a Behavior-Classification-model $\pi_{\mathrm{B}}, L(\delta \mid \pi)$ denote the likelihood of $\delta$ given a model $\pi$, and $\operatorname{size}(\pi)$ denote the size or complexity of a model $\pi$ (we define as the number of edges in the DBN of the model) then the BIC for an Action-model $\pi_{\mathrm{A}}$ is defined as

$$
\begin{gathered}
\log L\left(\delta \mid \pi_{A}\right)-\frac{\operatorname{size}\left(\pi_{A}\right)}{2} \cdot \log n \\
=\sum_{i=1}^{n}\left[\log P\left(\text { actions }^{i}, \text { actions }^{i-1} \text {, percepts }^{i} \mid \pi_{A}\right)\right]-\frac{\operatorname{size}\left(\pi_{A}\right)}{2} \cdot \log n
\end{gathered}
$$

and the BIC for a Behavior-Classification-model $\pi_{\mathrm{B}}$ is defined as

$$
\begin{gathered}
\log L\left(\delta \mid \pi_{B}\right)-\frac{\operatorname{size}\left(\pi_{B}\right)}{2} \cdot \log n \\
=\sum_{i=1}^{n}\left[\log P\left(\text { behavior }^{i} \text {,behavior }^{i-1} \text {, percepts }^{i} \mid \pi_{B}\right)\right]-\frac{\operatorname{size}\left(\pi_{B}\right)}{2} \cdot \log n .
\end{gathered}
$$

To focalize on the intended purpose of Action- and Behavior-Classificationmodels, we evolved a version of the BIC, which we refer as the Parent-Child BIC (PCh-BIC), where the likelihood is replaced by a parent-child-monitor [4]. Following the foregoing definition, the PCh-BIC for an Action-model $\pi_{\mathrm{A}}$ is defined as

$$
\sum_{i=1}^{n}\left[\log P\left(\text { actions }^{i} \mid \text { actions }^{i-1} \text { percepts }^{i}, \pi_{A}\right)\right]-\frac{\operatorname{size}\left(\pi_{A}\right)}{2} \cdot \log n
$$

while the PCh-BIC for a Behavior-Classification-model $\pi_{\mathrm{B}}$ is defined as

$$
\sum_{i=1}^{n}\left[\log P\left(\text { behavior }^{i} \mid \text { percepts }^{i}, \pi_{B}\right)\right]-\frac{\operatorname{size}\left(\pi_{B}\right)}{2} \cdot \log n .
$$

\subsubsection{Learning Procedure}

As the learning procedure of pertinent percepts doesn't differ between Action- and Behavior-Classification-models, it will be described for the learning of Action-models only: Starting with an initial Action-model $\pi_{\mathrm{A}}$ without any percepts (Fig. 3), new percepts are included in a step-wise manner.
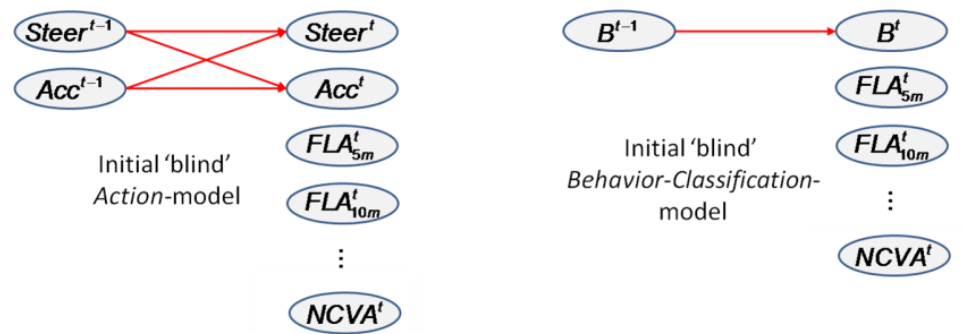

Fig. 3. DBN of an initial or blind Action-model and Behavior-Classification-model without any used percepts.

By now, a simple greedy-heuristic is used. For each one of the available percepts, 
Eilers, M. \& Möbus C., Learning the Relevant Percepts of Modular Hierarchical Bayesian Driver Models using a Bayesian Information Criterion, in: Vincent G. Duffy (Ed.), Digital Human Modeling, HCI 2011, Orlando, FL, USA

the PCh-BIC is calculated for the initial model extended by an edge from the actionvariable $A c c^{t}$ to the respective percept. Using the intended inference for longitudinal control $P\left(\right.$ Acc $^{t} \mid$ Acc $^{t-1}$, Steer $^{t-1}$, Percepts $\left.^{t}\right)$ as the parent-child-monitor, the PCh-BIC is calculated by:

$$
\sum_{i=1}^{n}\left[\log P\left(\operatorname{acc}^{i} \mid \operatorname{acc}^{i-1}, \text { steer }^{i-1}, f c a_{5 m}^{i}, \ldots, n c v a^{i}, \pi_{A}\right)\right]-\frac{\operatorname{size}\left(\pi_{A}\right)}{2} \cdot \log n .
$$

The percept leading to the best PCh-BIC (Fig. 4) can be seen as the most pertinent percept of the given possibilities for longitudinal control and is permanently included in the model.

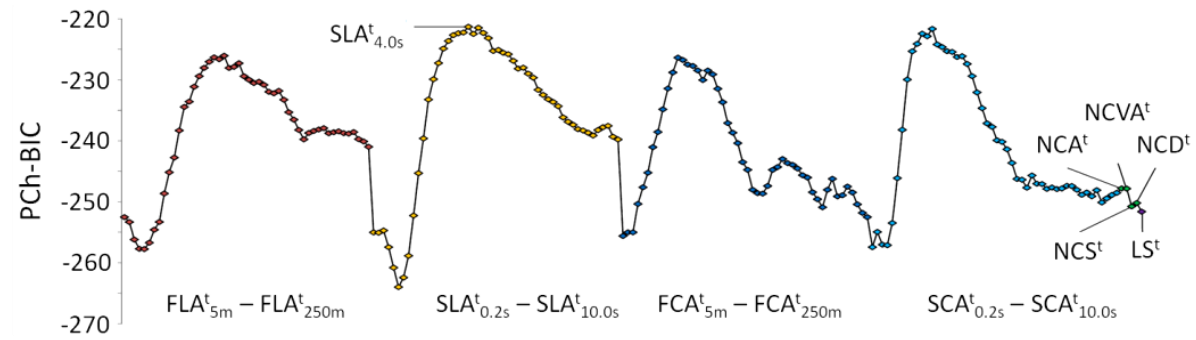

Fig. 4. Plot of PCh-BICs computed for an Action-model selecting one of 205 possible percepts for longitudinal control at a time. The PCh-BIC is maximized for a time-dependent percept $S L A_{4.0 s}^{t}$, revealing it as the most pertinent percept of the given possibilities for longitudinal control.

Next, for each of the remaining percepts, the PCh-BIC is calculated for the improved model extended by a new edge from the action-variable Steer ${ }^{t}$ to the respective percept. Using the intended inference for lateral control $P\left(\right.$ Steer $^{t} \mid$ Acc $^{t-1}$, Steer $^{t-1}$, Percepts $\left.^{t}\right)$ as the parent-child-monitor, the PCh-BIC is calculated by:

$$
\sum_{i=1}^{n}\left[\log P\left(\text { steer }^{i} \mid \text { acc }^{i-1}, \text { steer }^{i-1}, f c a_{5 m}^{i}, \ldots, n c v a^{i}, \pi_{A}\right)\right]-\frac{\operatorname{size}\left(\pi_{A}\right)}{2} \cdot \log n .
$$

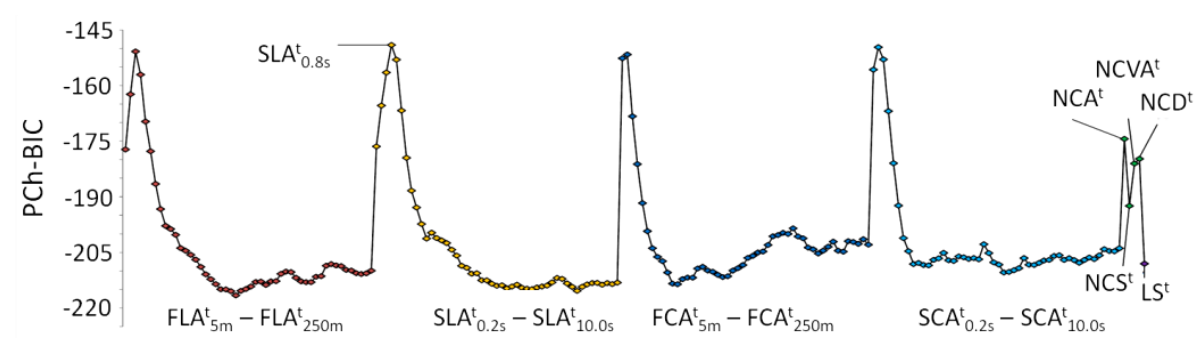

Fig. 5. Plot of PCh-BICs computed for an Action-model using one of 204 remaining possible percepts for lateral control at a time. The PCh-BIC is maximized for a time-dependent percept $S L A_{0.8 s}^{t}$, revealing it as the most pertinent percept of the given possibilities for lateral control.

The percept leading to the best PCh-BIC (Fig. 5) can be seen respectively as the 
Eilers, M. \& Möbus C., Learning the Relevant Percepts of Modular Hierarchical Bayesian Driver Models using a Bayesian Information Criterion, in: Vincent G. Duffy (Ed.), Digital Human Modeling, HCI 2011, Orlando, FL, USA

most pertinent percept of the given possibilities for lateral control and is likewise included permanently in the model.

The procedure will then be repeated with the new model. In this step-wise manner percepts are added until the PCh-BIC can't be improved any longer for any percept conditioned by $A c c^{t}$ or Steer ${ }^{t}$. As a result the learning procedure selects a minimal set of peephole percepts.

\section{Results and Discussion}

Using the learning procedure we revealed the most relevant peephole-percepts for all the nine Action-models and four Behavior-Classification-models of the skill hierarchy (Fig. 2). Learning the Action-models, 15 peephole percepts could be revealed as pertinent for longitudinal control, with the speed $L S^{t}$ and the time-independent percept $F L A^{t}{ }_{5 m}$ being the two most frequent ones (Table 2).

Table 2. Summary of the most relevant 15 peephole percepts used for longitudinal control.

\begin{tabular}{|c|c|c|c|}
\hline Nr. & Percept & Times used & Relevant for longitudinal control in the \\
\hline 1 & $F C A_{40 m}^{t}$ & 1 & PassCar Action-model \\
\hline 2 & $F C A_{80 m}^{t}$ & 1 & FollowSharp Action-model \\
\hline 3 & $F C A_{110 m}^{t}$ & 1 & FollowWide Action-model \\
\hline 4 & $F C A^{t}{ }_{135 m}^{110 m}$ & 1 & Sharp Action-model \\
\hline 5 & $F C A_{225 m}^{t}$ & 2 & FollowStraight and PassIn Action-model \\
\hline 6 & $F L A_{5 m}^{t}$ & 3 & Wide, Sharp and FollowWide Action-model \\
\hline 7 & $S C A_{0.4 s}^{t}$ & 1 & PassIn Action-model \\
\hline 8 & $S C A_{3.2 s}^{t}$ & 1 & Wide Action-model \\
\hline 9 & $S L A_{4.0 s}^{t}$ & 1 & Straight Action-model \\
\hline 10 & $S L A_{8.4 s}^{t}$ & 1 & FollowStraight Action-model \\
\hline 11 & $S L A_{9.0 s}^{t}$ & 1 & PassOut Action-model \\
\hline 12 & $\boldsymbol{L} \boldsymbol{S}^{t}$ & 4 & Straight, Sharp, FollowStraight and PassOut Action-model \\
\hline 13 & $N C A^{t}$ & 1 & FollowSharp Action-model \\
\hline 14 & $N C D^{t}$ & 1 & FollowWide Action-model \\
\hline 15 & $N C V A^{t}$ & 1 & FollowSharp Action-model \\
\hline
\end{tabular}

For lateral control 22 pertinent percepts could be revealed, with the timeindependent percept $F C A_{5 m}^{t}$ and the time-dependent percept $S L A_{0.8 s}^{t}$ being the most frequent ones (Table 3 ).

Table 3. Summary of the most relevant 22 peephole percepts used for lateral control.

\begin{tabular}{|c|c|c|c|}
\hline Nr. & Percept & Times used & Relevant for lateral control in the \\
\hline 1 & $F C A_{5 m}^{t}$ & 4 & Straight, Sharp, FollowStraight and PassOut Action-model \\
\hline 2 & $F C A_{65 m}^{t}$ & 1 & PassIn Action-model \\
\hline 3 & $F C A_{75 m}^{t}$ & 1 & Sharp Action-model \\
\hline 4 & $F C A_{175 m}^{t}$ & 1 & Sharp Action-model \\
\hline 5 & $F C A_{190 m}^{t}$ & 2 & Straight and FollowWide Action-model \\
\hline 6 & $F L A_{5 m}^{t}$ & 1 & PassOut Action-model \\
\hline 7 & $F L A_{10 m}^{t}$ & 1 & FollowSharp Action-model \\
\hline
\end{tabular}


Eilers, M. \& Möbus C., Learning the Relevant Percepts of Modular Hierarchical Bayesian Driver Models using a Bayesian Information Criterion, in: Vincent G. Duffy (Ed.), Digital Human Modeling, HCI 2011, Orlando, FL, USA

\begin{tabular}{|c|c|c|c|}
\hline 8 & $F L A_{15 m}^{t}$ & 1 & FollowWide Action-model \\
\hline 9 & $F L A_{75 m}^{t}$ & 1 & PassIn Action-model \\
\hline 10 & $F L A_{135 m}^{t}$ & 1 & FollowWide Action-model \\
\hline 11 & $F L A_{185 m}^{t}$ & 1 & Wide Action-model \\
\hline 12 & $F L A_{225 m}^{t}$ & 1 & FollowStraight Action-model \\
\hline 13 & $S L A_{0.2 s}^{t}$ & 2 & FollowWide and FollowSharp Action-model \\
\hline 14 & $S L A_{0.8 s}^{t}$ & 3 & Straight, Wide and Sharp Action-model \\
\hline 15 & $S L A_{1.2 s}^{t}$ & 1 & FollowSharp Action-model \\
\hline 16 & $S L A_{1.6 s}^{t}$ & 1 & Sharp Action-model \\
\hline 17 & $S L A_{2.0 s}^{t}$ & 1 & PassCar Action-model \\
\hline 18 & $S L A_{8.8 s}^{t}$ & 1 & PassOut Action-model \\
\hline 19 & $S L A_{9.6 s}^{t}$ & 1 & FollowSharp Action-model \\
\hline 20 & $S C A_{0.4 s}^{t}$ & 1 & Wide Action-model \\
\hline 21 & $S C A_{0.6 s}^{t}$ & 1 & FollowStraight Action-model \\
\hline 22 & $N C D^{t}$ & 1 & PassOut Action-model \\
\hline
\end{tabular}

Table 4 shows a summary of all used peephole percepts pertinent for classification of appropriate maneuvers or behaviors in the four Behavior-Classification-models.

Table 4. Summary of the most relevant 11 peephole percepts used for behavior-classification.

\begin{tabular}{|c|c|c|c|}
\hline Nr. & Percept & Times used & Relevant for classification of driving behavior in the \\
\hline 1 & $F C A_{30 m}^{t}$ & 1 & LaneFollowing Behavior-Classification-model \\
\hline 2 & $F C A_{60 m}^{t}$ & 1 & LaneFollowing Behavior-Classification-model \\
\hline 3 & $F C A_{205 m}^{t}$ & 1 & Racing Scenario Behavior-Classification-model \\
\hline 4 & $F L A_{5 m}^{t}$ & 2 & CarFollowing and Scenario Behavior-Classification-model \\
\hline 5 & $F L A_{200 m}^{t}$ & 1 & CarFollowing Behavior-Classification-model \\
\hline 6 & $F L A_{240 m}^{t}$ & 1 & CarFollowing Behavior-Classification-model \\
\hline 7 & $S C A_{0.2 s}^{t+16 m}$ & 1 & Overtaking Behavior-Classification-model \\
\hline 8 & $S C A_{0.6 s}^{t}$ & 2 & LaneFollowing and CarFollowing Behavior-Classification-model \\
\hline 9 & $S C A_{8.2 s}^{t}$ & 1 & LaneFollowing Behavior-Classification-model \\
\hline 10 & $N C A^{t}$ & 1 & Overtaking Behavior-Classification-model \\
\hline 11 & $N C D^{t}$ & 1 & Racing Scenario Behavior-Classification-model \\
\hline
\end{tabular}

Using only the 41 different peephole percepts that could be revealed during the learning process, the resulting $\mathrm{BAD}$ MoB model is able to drive on different racing courses in the TORCS simulation environment while overtaking slower vehicles (videos are available at http://www.lks.uni-oldenburg.de/46350.html).

For validation purposes we classed each of the models with a ranking of each five theoretical models of equal structure ranging from a totally uninformed model, solving the intended parent-child monitors (cf. (5) and (6)) with $P($ searched $\mid$ known) $=$ $1 /$ searched] (a probability equal to randomness), to a perfect model, solving it with $P($ searched $\mid$ known $)=1$ (a probability equal to certainty). Based on these rankings, the Racing Scenario Behavior-Classification-model is the best, while the Overtaking Behavior-Classification-model is the worst of the four Behavior-Classificationmodels, though all of them show very great results. The Action-models allow a greater space for future improvement, with the PassCar Action-model being the best and the Sharp Action-model being the worst of all nine Action-models. As a next step, the percepts obtained should be validated by experiments with human drivers [16]. 
Eilers, M. \& Möbus C., Learning the Relevant Percepts of Modular Hierarchical Bayesian Driver Models using a Bayesian Information Criterion, in: Vincent G. Duffy (Ed.), Digital Human Modeling, HCI 2011, Orlando, FL, USA

\section{References}

1. Bessière, P., Laugier, Ch., Siegwart, R. (eds.): Probabilistic Reasoning and Decision Making in Sensory-Motor Systems, Springer, Berlin (2008)

2. Cacciabue, P.C.: Modelling Driver Behaviour in Automotive Environments, Springer, London (2007)

3. Chattington, M., Wilson, M., Ashford, D., Marple-Horvat, D.E.: Eye-Steering Coordination in Natural Driving, Experimental Brain Research, 180, pp. 1--14 (2007)

4. Cowell, R.G., Dawid, A.Ph., Lauritzen, St.L., Spiegelhalter, D.J.: Probabilistic Networks and Expert Systems, Springer, Berlin (1999)

5. Eilers, M., Möbus, C.: Learning of a Bayesian Autonomous Driver Mixture-of-Behaviors (BAD-MoB) Model. In: Advances in Applied Digital Human Modeling, pp. 436--445, Duffy, V.G. (ed), CRC Press, Taylor \& Francis Group, Boca Raton, 2010/2011 (2010)

6. Eilers, M., Möbus, C.: Lernen eines modularen Bayesian Autonomous Driver Mixture-ofBehaviors (BAD MoB) Modells. In Jürgensohn, Th., Kolrep, H. (eds.), Fahrermodellierung in Wissenschaft und Wirtschaft, 3. Berliner Fachtagung für Fahrermodellierung, Fortschrittsbericht des VDI in der Reihe 22 (Mensch-MaschineSysteme), pp.61--74, VDI-Verlag (2010)

7. Horrey, W.J. et al.: Modeling Driver's Visual Attention Allocation While Interacting With In-Vehicle Technologies, J. Exp. Psych., 12, pp. 67--78 (2006)

8. Jensen, F.V., Nielsen, Th.D.: Bayesian Networks and Decision Graphs (2nd edition), Springer (2007)

9. Land, M.F.: The Visual Control of Steering. In L.R. Harris, M. Jenkin (eds.), Vision and Action, Cambridge: Cambridge University Press, pp. 163--180 (1998)

10. Land, M., Horwood, J.: Which Parts of the Road Guide Steering?, Nature, 377, pp.339-340 (1995)

11. Land, M., Lee, D.N.: Where we look when we steer, Nature, 369, pp.742--744 (1994)

12. Lee, D.N.: A theory of visual control of braking based on information about time-tocollision. Perception, 5, pp. 437--459 (1976)

13. Lee, D.N.: How Movement is Guided, http://www.perception-inaction.ed.ac.uk/PDF_s/Howmovementisguided.pdf (2005)

14. Möbus, C., Eilers, M.: Further Steps Towards Driver Modeling according to the Bayesian Programming Approach, in: Conference Proceedings, HCII 2009, Digital Human Modeling, pp. 413--422, LNCS (LNAI), Springer, San Diego (2009)

15. Möbus, C.,Eilers, M.: Mixture of Behaviors and Levels-of-Expertise in a Bayesian Autonomous Driver Model, in: Advances in Applied Digital Human Modeling, pp. 425-435, Duffy, V.G. (ed), CRC Press, Taylor \& Francis Group, Boca Raton, 2010/2011 (2010)

16. Möbus, C., Eilers, M., Garbe, H.: Prediction of Deficits in Situation Awareness with a Modular Hierarchical Bayesian Driver Model, HCII 2011 (submitted)

17. Pepping, G.J., Grealy, M.A.: Closing the Gap: The Scientific Writings of David N. Lee, Lawrence Erlbaum Associates, Publishers, Mahwah, N.J. (2007)

18. Schwarz, G.: Estimating the Dimension of a Model, The Annals of Statistics, Vol. 6, Nr. 2, pp. 461--464 (1978)

19. Wilkie R.M., Wann J.P.: Driving as night falls: The contribution of retinal flow and visual direction to the control of steering. Current Biology, 12, pp. 2014--2017 (2002)

20. Wilkie, R.M., Wann, J.P., Eye-Movements Aid the Control of Locomotion, Journal of Vision, 3, pp. 677--684 (2003)

21. Xu, Y., Lee, K.K.C.: Human Behavior Learning and Transfer. CRC Press, Boca Raton (2006) 